\title{
Simultaneous recovery of two ultrashort laser pulses from a single spectrogram
}

\author{
Kenneth W. DeLong and Rick Trebino \\ Sandia National Laboratories, MS 9057, Livermore, California 94551-0969 \\ William E. White \\ Lawrence Livermore Laboratories, Livermore, California 94550
}

Received January 17, 1995; revised manuscript received June 5, 1995

\begin{abstract}
We introduce a technique for simultaneously measuring the time-dependent intensity and phase of two independent and arbitrary ultrashort laser pulses from a single measured spectrogram. This two-pulse method is mathematically equivalent to the problem of blind deconvolution, and we use an algorithm analogous to those used for deblurring two-dimensional images to recover the two pulses. We demonstrate the method by simultaneously retrieving the intensity and the phase of two different pulses from a Ti:sapphire laser, one of which is chirped by propagation through glass. (C) 1995 Optical Society of America
\end{abstract}

It is not sufficient to be able to measure one ultrashort laser pulse; generally, one would like to be able to measure two such pulses simultaneously, for all ultrafastspectroscopy experiments involve at least one ultrashort pulse as input and, in addition, another such pulse as output, the measurement of both pulses being necessary to characterize the sample medium. Some ultrafastspectroscopic information is available if only the pulse energies are measured, but significantly more information is available if more complete characterization of the pulses is achieved. As a result, many researchers have expended much effort to characterize in greater detail both input and output pulses in material-characterization experiments. $^{1-3}$ For example, in four-wave-mixing experiments on semiconductors, Bigot et al. ${ }^{4}$ use an autocorrelation and a spectrum to characterize their input pulses and a variety of methods to extract intensity and phase information from their signal pulse. Indeed, the knowledge of the electric field of a pulse both before and after it passes through a medium determines the medium's absorption coefficient and refractive index for the entire bandwidth of the pulse. A technique for measuring two unknown ultrashort laser pulses would therefore be quite useful.

It has recently become possible to characterize one ultrashort laser pulse completely. ${ }^{5-8}$ This measurement is not so trivial that building two such devices is practical or cost effective. In addition, signal pulses are often too weak to be measured with these methods. It would be preferable to have a method that simultaneously yields the time-dependent intensity and phase of two independent pulses with a single apparatus and that allows one of the pulses to be weak.

In this paper we present and demonstrate such a method. Our method is a generalization of one of the above methods, frequency-resolved optical gating ${ }^{5,9-11}$ (FROG). In FROG, the pulse is used to gate itself, and the gated slice of the pulse is frequency resolved, gen- erating a spectrogram of the pulse. A phase-retrieval algorithm then extracts the pulse from the spectrogram. FROG can be described as a spectrally resolved autocorrelation. ${ }^{11}$ One could imagine attempting the two-pulse problem by performing spectrally resolved cross correlation, in which one pulse gates a different pulse. Unlike previous suggestions, in which complete a priori knowledge of one of the pulses is required, ${ }^{12}$ in our proposed method both pulses are presumed to be unknown. The pulse-extraction problem now is much more complex than in FROG, however, and it would not appear that such a trace contains sufficient information to determine both pulses. We take advantage of the fact that the mathematics that we encounter in the two-pulse-extraction problem is equivalent to twodimensional blind deconvolution, ${ }^{13}$ a technique from image science that quite counterintuitively allows one to extract both the image and an unknown blurring function from a blurred image. It has been shown that, although the one-dimensional blind-deconvolution problem has ambiguities, the two-dimensional version of this problem surprisingly yields essentially unique results, provided that a simple constraint, such as finite support or nonnegativity, exists. ${ }^{14}$ As a result, we use a modified blind-deconvolution algorithm that succeeds in extracting both pulses.

We call this method of retrieving the intensity and phase of both pulses from a frequency-resolved cross correlation $^{15}$ the twin recovery of electric-field envelopes by the use of FROG, or TREEFROG. ${ }^{15}$ In Fig. 1, we see the way that information about both the probe and the gate is contained in the TREEFROG trace. Figure 1 shows TREEFROG traces generated by a Gaussian probe pulse with temporal cubic phase and an unchirped gate pulse of various widths. Short gate pulses, which have a large spectral width, produce the TREEFROG traces that are narrow in the delay direction and wide in the frequency direction, whereas longer gate 

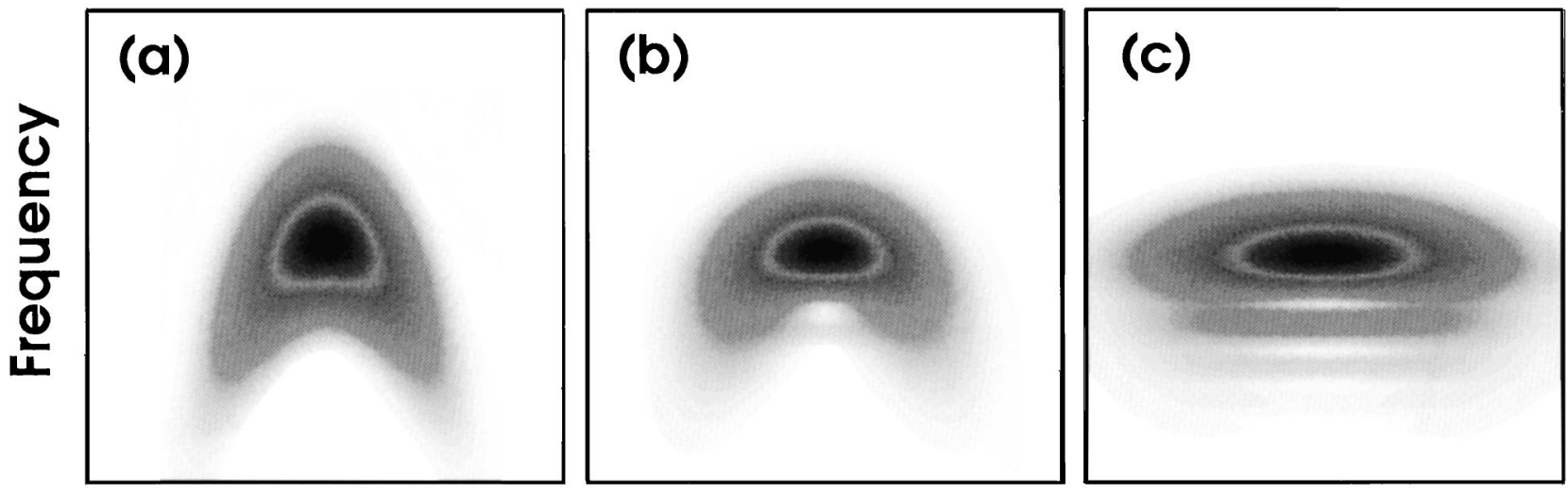

\section{Time}

Fig. 1. Second-harmonic generation TREEFROG traces generated by a probe with temporal cubic phase and an unchirped gate. Both pulses have a Gaussian intensity profile and were calculated on a 64-element array. The probe has a full-width at half-maximum of 10 , and the gate has a width of (a) 4, (b) 8, (c) 16 . As the gate gets smaller, its spectral content increases, so that the TREEFROG trace gets wider in the spectral dimension. The longer gate pulse (c), with its narrow spectrum, resolves spectral oscillations that are washed out in the traces made with shorter gate pulses.

pulses, which have a narrow spectrum, have the opposite effect.

In TREEFROG, we generate a signal field from the nonlinear mixing of two optical fields, which we write here, letting $P$ represent the probe and $G$ the gate, as

$$
E_{\text {sig }}(t, \tau)=P(t) G(t-\tau) .
$$

The nonlinear interaction in TREEFROG can be any fast nonlinearity. ${ }^{10}$ For example, in TREEFROG, with a polarization-gate beam geometry, $P(t)=E_{p}(t)$ and $G(t)=$ $\left|E_{g}(t)\right|^{2}$, where $E_{p}(t)$ and $E_{g}(t)$ are the electric fields of the probe and the gate, respectively. In this work, however, we use second-harmonic generation (SHG), which has been used to perform FROG measurements ${ }^{11,16,17}$ and that has $P(t)=E_{p}(t)$ and $G(t)=E_{g}(t)$. The TREEFROG trace is the magnitude squared of the spectrum of the signal field:

$$
I_{\text {TREEFROG }}(\omega, \tau)=\left|\int_{-\infty}^{\infty} \mathrm{d} t E_{\text {sig }}(t, \tau) \exp (i \omega t)\right|^{2} .
$$

The task of the pulse-retrieval algorithm is to find both $P(t)$ and $G(t)$ from $I_{\text {TREEFROG }}(\omega, \tau)$. Unfortunately, previous algorithms such as those used for FROG do not suffice. Fortunately, the mathematical problem represented by Eqs. (1) and (2) has been solved in an image science setting, in which it has been shown that this problem is equivalent to two-dimensional blind deconvolution, for which algorithms exist. ${ }^{14,18}$ The TREEFROG algorithm that we use is a modification of one such algorithm.

The TREEFROG algorithm begins with guesses for the fields $P(t)$ and $G(t)$ and generates $E_{\text {sig }}(\omega, \tau)$, Fourier transforming Eq. (1) with respect to $t$. On each iteration, we replace the magnitude of $E_{\mathrm{sig}}(\omega, \tau)$ with the square root of the experimentally measured TREEFROG trace intensity, but leave the phase unchanged, to yield a modified signal field $E_{\mathrm{sig}}^{\prime}(\omega, \tau)$. An inverse Fourier transform with respect to $\omega$ generates $E_{\mathrm{sig}}^{\prime}(t, \tau)$. We then use the method of generalized projections ${ }^{19-21}$ to generate new guesses for the fields. Specifically, we formulate an error function $Z$, as

$$
Z=\sum_{t, \tau=1}^{N}\left|E_{\mathrm{sig}}^{\prime}(t, \tau)-P(t) G(t-\tau)\right|^{2} .
$$

The implementation of generalized projections proceeds by modification of only one of the fields, $P(t)$ or $G(t)$, on a given iteration. ${ }^{18}$ On the even iterations we generate a new guess for $P(t)$ by minimizing $Z$ with respect to $P(t)$, and on the odd iterations we generate a new guess for $G(t)$ by minimizing $Z$ with respect to $G(t)$. The algorithm continues until the resulting TREEFROG trace matches the experimentally generated trace (or until the error between these two reaches a minimum).

In a series of numerical simulations, we found that using the spectra of the fields (an easily measured quantity) as an additional constraint improved the convergence of the TREEFROG algorithm considerably. On iterations in which one field is modified through the use of generalized projections, we also replace the spectrum of the other field with its measured spectrum just before the application of the generalized projection [the minimization of Eq. (3)]. Including spectral constraints in this manner removes potential ambiguities ${ }^{22}$ and appears to make the TREEFROG algorithm quite robust. Measurement of these spectra can be achieved easily with the same spectrometer and camera that record the TREEFROG trace and hence does not complicate the apparatus significantly. We also emphasize here that we make no assumptions regarding the pulses; the above algorithm is completely general.

We have demonstrated TREEFROG experimentally by using SHG as the nonlinearity in a multishot configuration (although a single-shot arrangement should be straightforward). The experimental setup is diagrammed in Fig. 2. A beam from a Spectra-Physics Tsunami Ti:sapphire laser oscillator operating at $757 \mathrm{~nm}$ was split into two beams, a probe and a gate. The gate beam was passed through a variable time delay and a 6.5-cm length of BK7 glass. The two beams were then focused into a KDP frequency-doubling crystal, and the 
sum-frequency light was frequency resolved by a spectrometer. Recording this spectrum for all relevant delays between the two beams resulted in the TREEFROG trace seen in Fig. 3. Unlike SHG FROG, ${ }^{10,11,17}$ the SHG TREEFROG trace acquires a tilt from chirp, because the probe and the gate fields are different.

We were able to retrieve the time-dependent intensity and phase of both the gate and the probe fields by using the TREEFROG algorithm described above. As propagation through BK7 glass leaves the spectrum of this pulse unchanged, we used the same spectrum to constrain both the probe and the gate fields. Also, because inevitable noise in the measured spectrum caused the spectrumconstraining process to introduce excess noise into the fields, after the algorithm reached what appeared to be its lowest obtainable error (after 100 iterations) we performed six additional iterations of the algorithm without the spectral constraint. This served to reduce the noise in the retrieved fields. The final error $G$ (as defined in Ref. 17) was 0.00194 (on a $128 \times 128$ pixel trace).

The retrieved fields are shown in Fig. 4. To verify these fields, we first independently made SHG FROG traces ${ }^{17}$ of the oscillator beam with and without the BK7 present in order to determine the intensity and the phase of each field separately for comparison with the TREEFROG result. Figures 4(a) and 4(b) show the probe and the gate fields, respectively, each measured

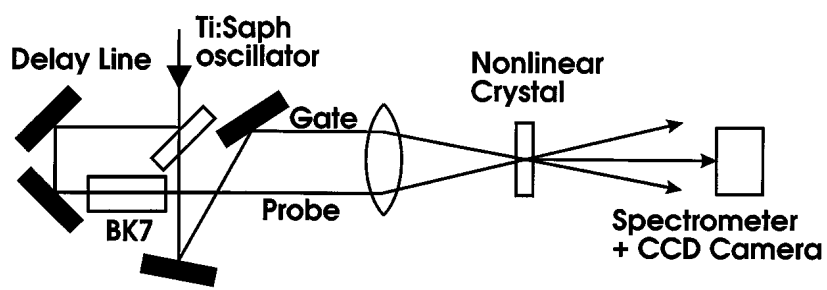

Fig. 2. Experimental arrangement for SHG TREEFROG. The probe pulse is chirped by propagation through $6.5 \mathrm{~cm}$ of $\mathrm{BK} 7$ glass. The cross-correlation signal generated through SHG is frequency resolved to yield the TREEFROG trace.

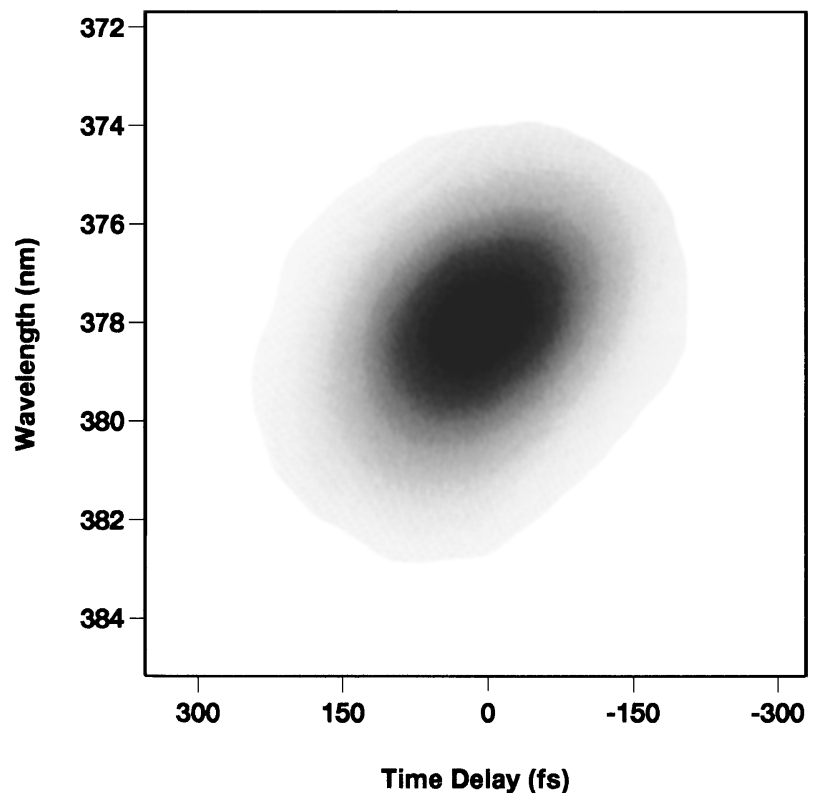

Fig. 3. Experimentally measured TREEFROG trace.
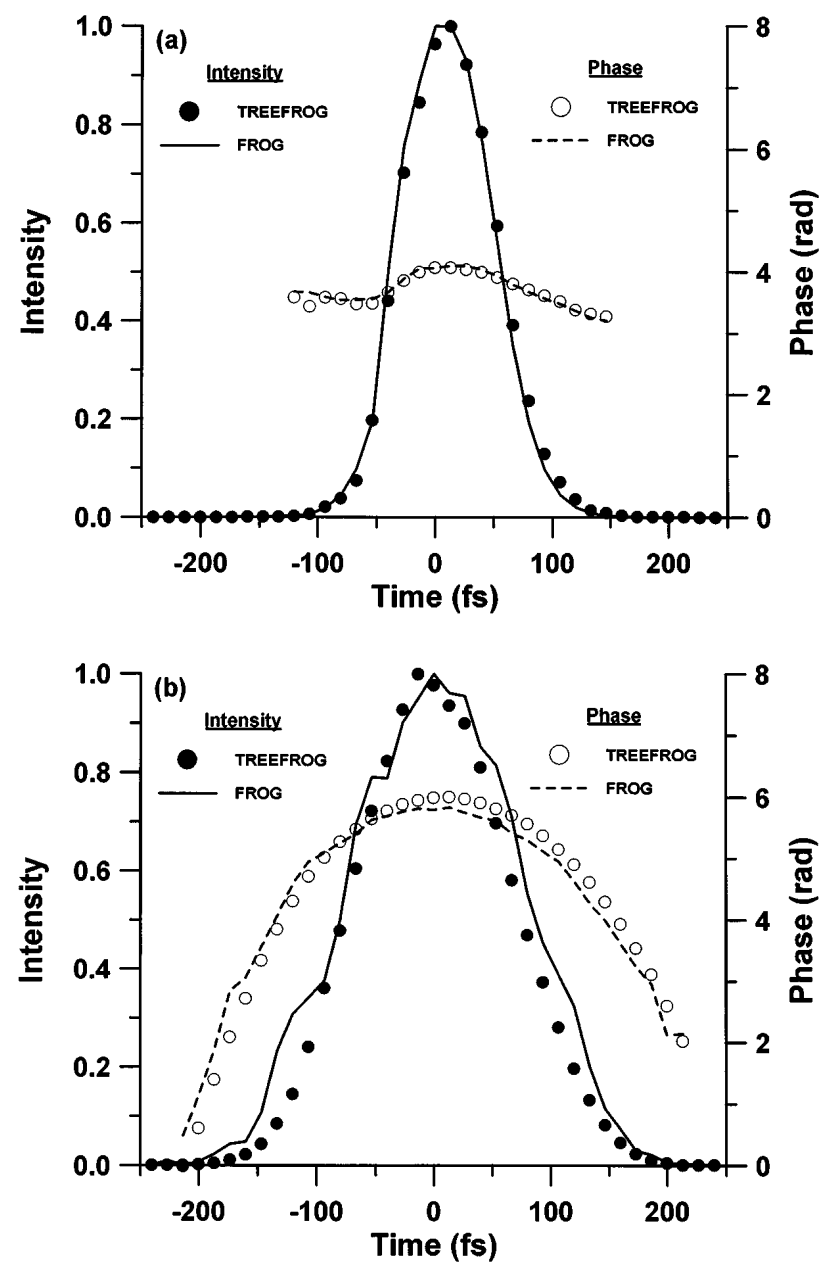

Fig. 4. Fields of the (a) probe and (b) gate retrieved by the use of TREEFROG compared with the fields retrieved by the use of standard SHG FROG. The agreement is quite good.

with TREEFROG and independently with FROG. The quite remarkable agreement between the fields retrieved with TREEFROG and the fields derived with SHG FROG indicates that the TREEFROG algorithm was quite successful at retrieving the intensity and phase of both the probe and the gate fields.

We can use the TREEFROG results to measure the group-velocity dispersion of BK7 glass. By performing a quadratic fit to the phases of the TREEFROG-derived fields in Fig. 4 over the wavelength range $745-770 \mathrm{~nm}$, we can calculate the dispersion parameter $\beta_{2}$ to be $393 \mathrm{fs}^{2} / \mathrm{cm}$ for BK7 glass. ${ }^{23}$ The actual value of $\beta_{2}$ for BK7 is $487 \mathrm{fs}^{2} / \mathrm{cm}^{24}$ The value obtained by a similar calculation from the phases of the two FROG-derived fields yield $490 \mathrm{fs}^{2} / \mathrm{cm}$. In this case, the FROG-derived phase is closer to the correct value than the TREEFROG-derived phase is. However, there is no fundamental reason that this should be so. Given proper convergence of the algorithm, TREEFROG should have the same flexibility as FROG in measuring phases, including higher-order and other complicated phases. The ability of FROG to retrieve the proper phase from a noisy trace has been well documented $^{25}$; TREEFROG is expected to have similar accuracy.

In conclusion, we have proposed and demonstrated a method we call TREEFROG for simultaneously measur- 
ing the intensity and the phase of two independent ultrashort laser pulses. TREEFROG should operate well with all FROG geometries ${ }^{10}$ and should have a variety of applications in experiments that require full characterization of both input and output pulses, even when one of the pulses is relatively weak.

\section{ACKNOWLEDGMENTS}

R. Trebino and K. DeLong acknowledge the support of the U.S. Department of Energy, Offices of Basic Energy Sciences, Chemical Sciences Division. The authors thank James Hunter for assistance in the laboratory.

\section{REFERENCES AND NOTES}

1. A. Freiberg and P. Saari, "Picosecond spectrochronography," IEEE J. Quantum Electron. QE-19, 622-630 (1983).

2. M. Mycek, S. Weiss, J. Bigot, S. Schmitt-Rink, and D. S. Chemla, "Femtosecond time-resolved free-induction decay of room-temperature exitons in GaAs quantum wells," Appl. Phys. Lett. 60, 2666-2668 (1992).

3. V. G. Lyssenko, J. Erland, I. Balslev, K.-H. Pantke, B. S. Razbirin, and J. M. Hvam, "Nature of nonlinear four-wavemixing beats in semiconductors," Phys. Rev. B 48, 57205723 (1993)

4. J.-Y. Bigot, M.-A. Mycek, S. Weiss, R. G. Ulbrich, and D. S. Chemla, "Instantaneous frequency dynamics of coherent wave mixing in semiconductor quantum wells," Phys. Rev. Lett. 70, 3307-3310 (1993).

5. D. J. Kane and R. Trebino, "Single-shot measurement of the intensity and phase of an arbitrary ultrashort pulse by using frequency-resolved optical gating," Opt. Lett. 18, 823-825 (1993).

6. J. L. A. Chilla and O. E. Martinez, "Direct determination of the amplitude and the phase of femtosecond light pulses," Opt. Lett. 16, 39-41 (1991).

7. K. C. Chu, J. P. Heritage, R. S. Grant, K. X. Liu, A Dienes, W. E. White, and A. Sullivan, "Direct measurement of the spectral phase of femtosecond pulses," Opt. Lett. 20, 904-906 (1995).

8. B. S. Prade, J. M. Schins, E. T. J. Nibbering, M. A. Franco, and A. Mysyrowicz, "A simple method for the determination of the intensity and phase of ultrashort optical pulses," Opt. Commun. 113, 79-84 (1995).

9. R. Trebino and D. J. Kane, "Using phase retrieval to measure the intensity and phase of ultrashort pulses: frequencyresolved optical gating," J. Opt. Soc. Am. A 10, 1101-1111 (1993).
10. K. W. DeLong, R. Trebino, and D. J. Kane, "A comparison of ultrashort-pulse frequency-resolved-optical-gating traces for three common beam geometries," J. Opt. Soc. Am. B 11, 1595-1608 (1994).

11. J. Paye, M. Ramaswamy, J. G. Fujimoto, and E. P. Ippen, "Measurement of the amplitude and phase of ultrashort light pulses from spectrally resolved autocorrelation," Opt. Lett. 18, 1946-1948 (1993).

12. J. Paye, "The chronocyclic representation of ultrashort light pulses," IEEE J. Quantum Electron. 28, 2262-2273 (1992).

13. B. C. McCallum and J. M. Rodenburg, "Simultaneous reconstruction of object and aperture functions from multiple far-field intensity measurements," J. Opt. Soc. Am. A 10, 231-239 (1993).

14. G. R. Ayers and J. C. Dainty, "Iterative blind deconvolution method and its applications," Opt. Lett. 13,547-549 (1988).

15. K. W. DeLong and R. Trebino, "Measuring the intensity and phase of two ultrashort pulses on a single shot," in Generation, Amplification, and Measurement of Ultrashort Laser Pulses, R. P. Trebino and I. A. Walmsleys, eds., Proc. Soc. Photo-Opt. Instrum. Eng. 2116, 268-274 (1994).

16. Y. Ishida, K. Naganuma, and T. Yajima, "Self-phase modulation in hybridly mode-locked CW dye lasers," IEEE J. Quantum Electron. 21, 69-77 (1985).

17. K. W. DeLong, R. Trebino, J. Hunter, and W. E. White, "Frequency-resolved optical gating using second-harmonic generation," J. Opt. Soc. Am. B 11,2206-2215 (1994).

18. J. H. Seldin and J. R. Fienup, "Interative blind deconvolution algorithm applied to phase retrieval," J. Opt. Soc. Am. A 7, 428-433 (1990).

19. A. Levi and H. Stark, Image Recovery: Theory and Applications, H. Stark, ed. (Academic, San Diego, 1987), pp. $277-320$.

20. K. W. DeLong, D. N. Fittinghoff, R. Trebino, B. Kohler, and K. Wilson, "Pulse retrieval in frequency-resolved optical gating using the method of generalized projections," Opt. Lett. 19, 2152-2154 (1994).

21. Y. Yang, N. P. Galatsanos, and H. Stark, "Projection-based blind deconvolution,” J. Opt. Soc. Am. A 11, 2401-2409 (1994).

22. In particular, pairs of linearly chirped Gaussian pulses exhibit ambiguities without the spectral constraint. It is unknown whether these ambiguities represent a general feature of TREEFROG or occur only for these pulses.

23. G. P. Agrawal, Nonlinear Fiber Optics (Academic, San Diego, 1989), p. 8.

24. Schott Computer Glass Catalog 1.0, Schott Glasswerke, Mainz, Germany, 1992.

25. D. N. Fittinghoff, K. W. DeLong, R. Trebino, and C. L. Ladera, "Noise sensitivity in frequency-resolved optical gating measurements of ultrashort pulses," J. Opt. Soc. Am. B 12, 1955-1967 (1995) 